\title{
Otimização de uma carteira de ativos setoriais utilizando o modelo Black-Litterman
}

Renato Cesar Sato,

Antonio A. Chaves
Luiz L. Salles-Neto,

\author{
João L. Chela
}

Departamento de Ciência e Tecnologia, ICT - UNIFESP
13084-790, São José dos Campos, SP

\author{
Email: rcsato@unifesp.br \\ luiz.leduino@gmail.com \\ antoniochaves@gmail.com \\ chela.risco@gmail.com
}

\begin{abstract}
Resumo: Nesse artigo foram utilizados seis conjuntos de ações de empresas de aviação negociadas na bolsa de valores de Nova Iorque (NYSE). As empresas selecionadas arbitrariamente tiveram como objetivo compor uma carteira setorial para aplicação do modelo Black-Litterman. As vantagens dessa aplicação são a superação da sensibilidade das mudanças nos valores esperados no modelo de Markowitz e a possibilidade de inclusão da visão do analista no modelo. Na primeira parte é apresentado brevemente o modelo Black-Litterman e, em seguida, são apresentados os dados utilizados na análise e os resultados obtidos. O modelo Black-Litterman mostrou-se com baixo desvio padrão em relação aos retornos históricos dos ativos.
\end{abstract}

\section{Introdução}

Parte dos trabalhos de finanças quantitativas são relacionados ao gerenciamento do risco de uma carteira. A abordagem mais comum é a otimização da carteira através da composição dos seus ativos. Essa talvez seja a principal motivação da teoria financeira: confrontar o problema entre o risco e o retorno.

Nesse trabalho buscamos complementar essa visão de otimização através das alocações dos ativos de uma carteira de investimentos realizadas com base na sinalização do mercado utilizando modelo de Black-Litterman [2], [3], [4], [5]. Esse modelo permite realizar uma otimização reversa para chegar a um equilíbrio do excesso de retornos de uma carteira de investimento. Podemos entender como excesso de retorno a diferença do retorno efetivo da carteira em relação a um outro ativo. Outro atrativo desse modelo é oferecer alternativas para os problemas encontrados com o modelo de Markowitz [9] [10] que considera as carteiras altamente concentradas e possui grande sensibilidade para pequenas mudanças nos valores esperados. É reconhecido que Markowitz mudou o paradima do gerenciamento dos investimentos abrindo caminho para uma série de implementações estatísticas sobre como alocar os ativos em uma carteira de investimentos. No entanto, não é raro obtermos através desses modelos resultados inadequados acerca das alocações dos ativos na carteira. A estrutura mecanicista da otimização da carteira pode levar a péssimas previsões quando utilizamos dados históricos dos retornos de um ativo. Isso é decorrente da estimação da covariância entre os retornos dos ativos e a estimação dos retornos esperados com base em dados históricos que podem produzir números pouco aderentes a realidade, como por exemplo, valores distanciados da média histórica dos retornos de um ativo ou carteira de investimentos [1].

A moderna teoria das carteiras de investimento assume que os investidores são avessos ao risco, isso significa que diante de dois ativos com o mesmo retorno esperado, entendendo como retorno esperado a média da distribuição probabilística dos retornos possíveis. Para um investidor somente aceitará um maior risco se houver um excesso de retorno em relação ao 
nível anterior de retorno esperado. Desse modo, assumimos que esse excesso de retorno implica em um maior risco em relação a um outro ativo. Nesse texto utilizamos como comparação do excesso de retornos, a diferença dos ativos selecionados com o índice S\&P 500 que nesse estudo terá o papel de representar o retorno do mercado.

O modelo Black-Litterman inicialmente foi proposto como meio de definir as alocações dos ativos, porém ele pode ser utilizado para previsão dos retornos esperados. O modelo inicia com a premissa que um investidor escolhe sua carteira ótima valendo-se de um dado grupo de ativos. A partir dessa escolha o modelo toma como ponto de partida a premissa de que, na ausência de informações adicionais, a comparação não pode ser melhorada [8]. De maneira resumida, ao invés de inserir os dados e a partir deles derivar uma carteira ótima, o modelo assume que uma dada carteira é ótima e valendo-se dessa premissa deriva os retornos esperados dos componentes. Assim, o vetor implícito dos retornos esperados é o ponto de partida do modelo. Com a introdução do modelo Black-Litterman foi possível os investidores adicionarem sua visão (opinião) na tomada de decisão relativa a alocação dos ativos em uma carteira. Vale ainda ressaltar que apesar do trabalho de Lee [7] a literatura acadêmica sobre a alocação tática de ativos ainda é relativamente dispersa. Desse modo, esse trabalho busca contribuir nos seguintes aspectos: 1) mostrar como a visão do analista pode ser incluída na modelagem de uma carteira de investimentos; 2) oferecer uma abordagem alternativa ao Modelo de Precificação de Ativos de Capital (Capital asset pricing model - CAPM) na alocação tática de ativos para formação de uma carteira de investimentos; 3 ) compor uma carteira setorial de ativos oriundos de empresas ligação ao ramo da aviação civil como exemplo de aplicação dessa abordagem teórica.

Na próxima seção é apresenta formalmente a estrutura do modelo.

\section{Estrutura do Modelo}

O modelo utiliza duas principais fontes para fornecer os resultados esperados: A primeira diz respeito sobre o que o mercado sugere a respeito dos excessos de retornos esperados; e a segunda esta relacionada a visão (opinião) que o investidor tem a respeito de um conjunto de ativos. A vantagem do modelo Black-Litterman é combinar diferentes fontes para estimação dos resultados esperados e com isso superar as limitações do modelo de otimização tradicional da variância média, que é sensível às variações dos retornos esperados. Desse modo, o modelo Black-Litterman sugere um ponto de partida neutro, e o equilíbrio implicíto dos retornos do mercado são expressos na forma de um vetor que deve satisfazer a relação [3]:

$$
\Pi=\lambda \Sigma \omega_{m k t}
$$

Onde, П é o retorno implicíto do equilíbrio de mercado ( $\mathrm{N}$ x 1); $\lambda$ é o parâmetro de aversão ao risco (sendo $\lambda>0) ; \Sigma$ é a matriz de variância-covariância dos retornos $(\mathrm{N} \times \mathrm{N})$ e $\omega_{m k t}$ são os pesos do equilíbrio do mercado ( $\mathrm{N}$ x 1$)$.

Esse retorno implicíto incorporado juntamente com a visão do investidor é a diferença oferecida por essa abordagem. As visões dos investidores podem ser de duas naturezas: relativa ou absolutas. As visões absolutas são difíceis de serem sustentadas no mercado financeiro, e, portanto, o uso das visões relativas são comumente utilizadas. Um exemplo de uma visão absoluta é dizer que o S\&P 500 será de $10 \%$ ao ano em 2014; ao passo que uma visão relativa diz que as ações da empresa AAA irão superar as ações da empresa BBB em $3 \%$ no ano de 2014. Colocado essas posições, o investidor inclui no modelo um vetor $\mathrm{Q}$ ( $\mathrm{K} \times 1$ ), onde $\mathrm{K}$ representa o número de visões. Na prática, os analistas podem ter maior facilidade e habilidade de fazer previsões sobre uma determinada classe de ativos em relação a outra classe. Para incluir no modelo essa limitação de visão do investidor é adicionado um vetor de erro ao vetor das visões $\mathrm{Q}$. 


$$
Q+\varepsilon=\left[\begin{array}{c}
Q_{1} \\
\vdots \\
Q_{k}
\end{array}\right]+\left[\begin{array}{c}
\varepsilon_{1} \\
\vdots \\
\varepsilon_{k}
\end{array}\right]
$$

A variação na incerteza expressa é através do vetor de erros. Essa variância de cada termo de erro forma uma nova matriz expressa por $\Omega$. Os elementos fora da diagonal de $\Omega$ expressam a covariância entre as visões. Na aplicação do modelo realizada nesse texto para fins de simplicação consideramos essa correlação igual a zero.

A matriz $\mathrm{P}$ representa a relação entre quais ativos o investidor possui suas visões. Essa matriz $(\mathrm{K} x \mathrm{~N})$ é expressa por:

$$
P=\left(\begin{array}{ccc}
P_{1,1} & \cdots & P_{1, N} \\
\vdots & \ddots & \vdots \\
P_{K, 1} & \cdots & P_{K, N}
\end{array}\right)_{K \times N}
$$

Assumindo que $P \times E(r)=Q+\varepsilon$ são normalmente distribuídos e adicionando os retornos implicítos do equilíbrio na equação completa de Black-Litterman:

$$
E(r)=\left[(\tau S)^{-1}+P^{T} \Omega^{-1} P\right]^{-1}\left[(\tau S)^{-1} \Pi+P^{T} \Omega^{-1} Q\right]
$$

Onde, $\tau$ é um valor escalar, e quanto maior esse valor mais enfâse é dado no equilíbrio e menos nas visões - nesse texto utilizamos o valor de 1 para fins de neutralidade nesse aspecto; $\mathrm{S}$ a matriz de variância-covariância para todos os ativos da carteira em análise; $\Omega$ a incerteza inerente a visão do investidor; $\Pi$ os excessos de retornos implícitos; $Q$ as visões sobre os excessos de retornos para alguns ou todos os ativos e $\mathrm{P}$ a matriz de ligação que representa quais os ativos o investidor possui visões.

As estimativas do modelo Black-Litterman são baseadas na otimização reversa para resolver o excesso de retorno ao invés dos pesos dos ativos utilizados na composição da carteira e na visão do analista sobre os ativos, ou seja, a confiança na sua visão. Essa estrutura de visão pode ser facilmente transportada para outros ativos do mercado como índices, ações etc.

\section{Dados Utilizados}

Para estimação de uma carteira de investimento setorial foram selecionados as ações de seis empresas relacionadas com o mercado de aviação. Essa seleção ocorreu de maneira deliberada, servindo como exemplo da aplicação do modelo em de modo alternativo a outros modelos precificação de ativos, como o modelo de precificação de ativos de capital (Capital Asset Pricing Model - CAPM). As empresas selecionadas foram Embraer (ERJ), Lookhead (LMT), Boeing (BA), American Airlines (AAL), Delta Airlines (DAL), United Airlines (UAL). Os valores de fechamento foram obtidos na Bolsa de Valores de Nova Iorque (New York Stock Exchange - NYSE). Para estimação do modelo foram selecionados valores de fechamento no período compreendido entre Junho de 2008 até Janeiro de 2014, totalizando 68 observações para cada empresa, ou seja, 408 observações no total. Como taxa livre de risco para cálculo do excesso de retorno utilizamos a Treasury Bill (T-Bill) mensal dos Estados Unidos.

Para obter os pesos da carteira consideramos que as seis ações selecionadas oferecem uma representação do mercado. Essa foi uma simplificação necessária para evitar a complexidade de achar os pesos da carteira.

\section{Resultados}

Baseados nos dados obtidos dos valores de fechamento mensal de cada empresa, do T-Bill mensal como taxa livre de risco, dos valores mensais do índice SP\&500 e do valor de capitalização de cada empresa na bolsa de valores obtivemos os seguintes resultados: 
Considerando que as ações selecionadas representam o universo na totalidade, conforme apresentado na Tabela 1, os pesos obtidos foram derivados do tamanho da capitalização no mercado de cada empresa. Os dados da capitalização dizem respeito ao valor estipulado no mês de fevereiro de 2014 na Bolsa de Valores de Nova Iorque.

A capitalização de mercado expressa por Mak.Cap diz respeito ao total de ações emitidas em dólares das empresas de capital aberto utilizadas nesse estudo. O termo Mak.Wt é o peso ponderado de cada ativo na carteira, isto é, a participação relativa em termos da capitalização de mercado.

\begin{tabular}{ccc}
\hline Capitalização do Mercado (US\$ Bi) & Mak.Cap & Mak.Wt \\
\hline ERJ & 6,21 & 0,03 \\
LMT & 53,50 & 0,25 \\
BA & 96,30 & 0,45 \\
AAL & 12,17 & 0,06 \\
DAL & 27,22 & 0,13 \\
UAL & 16,50 & 0,08 \\
Total & 211,90 & 1 \\
\hline
\end{tabular}

Tabela 1 - Valor de capitalização no mercado das empresas (em bilhões de dólares)

\begin{tabular}{lllllll}
\hline \multicolumn{1}{c}{ ERJ } & \multicolumn{1}{c}{ LMT } & \multicolumn{1}{c}{ BA } & AA & \multicolumn{1}{c}{ DAL } & \multicolumn{1}{c}{ UAL } \\
\hline ERJ & 0,012954447 & 0,005019077 & 0,006173386 & 0,011365559 & 0,006175171 & 0,01018503 \\
LMT & 0,005019077 & 0,005101809 & 0,004269152 & 0,005539277 & 0,00208186 & 0,00384006 \\
BA & 0,006173386 & 0,004269152 & 0,006980975 & 0,007564234 & 0,004435437 & 0,007416701 \\
AA & 0,011365559 & 0,005539277 & 0,007564234 & 0,060350651 & 0,029142763 & 0,040498529 \\
DAL & 0,006175171 & 0,00208186 & 0,004435437 & 0,029142763 & 0,022563074 & 0,021759988 \\
UAL & 0,01018503 & 0,00384006 & 0,007416701 & 0,040498529 & 0,021759988 & 0,040317702 \\
\hline
\end{tabular}

Tabela 2 - Matriz de variância-covariância das ações.

Para estimação da aversão do risco do investidor utilizamos as cotações do SP\&500 para estimar o excesso de retorno do mercado e a variância dos retornos do mercado através dos logretornos. Esse valor foi obtido pela razão entre o excesso de retorno do mercado, representando pelo retorno esperado $E\left(r_{m}\right)$ menos a taxa livre de risco $r_{f}$ dividido pela variância dos retornos do mercado.

$$
A=\frac{E\left(r_{m}\right)-r_{f}}{\sigma_{m}^{2}}=\frac{-0,0109}{0,002753}=-3,9431
$$

O excesso de retorno implícito seguiu a aplicação da aversão do risco estimado, a matriz de variância-covariância e pesos para estabelecimento do vetor (П).

Na Tabela 3, a equação R=ASW representa o excesso de retorno do equilíbrio implícito onde A é o preço do risco acima definido; S é a matriz de variância-covariância (Tabela 2) e W são os pesos ponderados definidos como Mak.Wt (Tabela 1).

\begin{tabular}{cc}
\hline Empresa & $(\mathrm{R}=\mathrm{ASW})$ \\
\hline ERJ & $-0,03$
\end{tabular}




\begin{tabular}{cc} 
LMT & $-0,02$ \\
BA & $-0,02$ \\
AAL & $-0,06$ \\
DAL & $-0,04$ \\
UAL & $-0,05$ \\
\hline
\end{tabular}

Tabela 3 - Excesso de Retorno do Equilíbrio Implicíto

As visões expressas na Tabela 4 foram definidas de maneira arbitrária, para demonstrar o papel do analista na definição dos parâmetros do modelo conforme comentado inicialmente nesse texto.

\begin{tabular}{cccccccc}
\hline Visão & Q & ERJ & LMT & BA & AAL & DAL & UAL \\
\hline Visão 1 & 0,005 & 1 & -1 & 0 & 0 & 0 & 0 \\
Visão 2 & 0,01 & -1 & 0 & 1 & 0 & 0 & 0 \\
Visão 3 & 0,005 & 0 & 0 & 0 & 0 & 1 & -1 \\
\hline
\end{tabular}

Tabela 4 - Visão e Matriz de Ligação

A maneira mais fácil para calibrar o modelo é através das premissas feitas para o valor do escalar $\tau$ que nesse texto assumimos igual a um. A literatura sobre o valor ideal para esse escalar não é conclusiva, e, podemos perceber que como a incerteza da média é menor do que a incerteza do retorno esse valor se aproxima de zero [3, 6]. Sendo $\Omega=\tau P S P^{T}$, a visão das incertezas, onde P é a matriz de ligação (Tabela 4), e $\mathrm{S}$ a matriz de variação-covariação dos retornos dos ativos (Tabela 2), podemos escrever na forma de matriz:

$$
\Omega=\left(\begin{array}{ccc}
0,008 & -0,006 & -0,002 \\
-0,006 & 0,008 & 0,001 \\
-0,002 & 0,001 & 0,019
\end{array}\right)
$$

A matriz $\Omega$ é uma matriz do tipo 3 X 3 devido ao número de visões no modelo e seus valores dizem respeito a incerteza associadas com as visões estabelecidas.

Para fins de comparação é apresentado na Tabela 5 uma comparação entre os resultados obtidos pelo modelo e a média dos retornos históricos no período. O modelo Black Litterman (BL) mostrou uma razoavél aproximação dos dados históricos, sendo um pouco mais conservador nos retornos esperados.

\begin{tabular}{cccc}
\hline Empresa & BL & Média Histórica & Desvio Padrão \\
\hline ERJ & $-0,021$ & $-0,012$ & $1 \%$ \\
LMT & $-0,019$ & $-0,006$ & $1 \%$ \\
BA & $-0,015$ & $-0,004$ & $1 \%$ \\
AAL & $-0,054$ & 0,023 & $5 \%$ \\
DAL & $-0,028$ & 0,009 & $3 \%$
\end{tabular}




\begin{tabular}{llll} 
UAL & $-0,038$ & 0,017 & $4 \%$ \\
\hline
\end{tabular}

Tabela 5 - Retorno Esperado do Modelo

\section{Conclusão}

O modelo de Black-Litterman mostrou-se adequado como alternativa para modelagem de uma carteira de investimentos setoriais quando comparado com a média histórica. A possibilidade do analista incluir suas visões faz com que o modelo seja atrativo para superar limitações da estrutura de alocação de ativos proposta por Markowitz, como por exemplo carteiras altamente concentradas, alta sensibilidade e problemas na estimação da maximização dos erros. Outra vantagem é a possibilidade do analista escalonar os níveis de confiança das visões em termos relativos, sendo esse o caso mais frequente encontrado na prática. Como sugestões para trabalhos futuros propomos a inclusão de uma analise das séries temporais e suas distribuições a priori e posteriori, bem como a comparação com outros modelos para o cálculo dos retornos esperados.

\section{Referências}

[1] Benninga S. Financial modeling. MIT press, Cambridge, 2008.

[2] Black F, and Litterman R. Global asset allocation with equities, bonds, and currencies. Fixed Income Research, vol. 2, pp 15-28, (1991).

[3] Black F, and Litterman R. Global portfolio optimization. Financial Analysts Journal, vol.48, pp. 28-43, (1992).

[4] Black F, and Litterman R. Asset allocation: combining investor views with market equilibrium. The Journal of Fixed Income, vol 1, pp. 7-18, (1991).

[5] He G. The intuition behind Black-Litterman model portfolios. Goldman Sachs Investment Management Series, 1999.

[6] Idzorek T.M. A step-by-step guide to the Black-Litterman model. Forecasting expected returns in the financial markets, in Forecasting expected returns in the financial markets. Academic Press, 2011.

[7] Lee W. Theory and Methodology of Tactical Asset Allocation. John Wiley \& Sons, 2000.

[8] Malkiel B.G. Reflections on the efficient market hypothesis: 30 years later. Financial Review vol. 40, pp. 1-9, (2005).

[9] Pfaff B. Financial Risk Modelling and Portfolio Optmization with R. John Wiley \& Sons, 2013.

[10] Ross S; Westerfield R; Jaffe J. Corporate Finance, $9^{\text {th }}$ edition, McGraw-Hill, 2009. 